\title{
A simple method for fabricating multi-layer PDMS structures for 3D microfluidic chips
}

\author{
Mengying Zhang, ${ }^{a}$ Jinbo Wu, ${ }^{a}$ Limu Wang, ${ }^{a}$ Kang Xiao ${ }^{b}$ and Weijia Wen ${ }^{* a b}$ \\ Received 4th November 2009, Accepted 13th January 2010 \\ First published as an Advance Article on the web 9th February 2010 \\ DOI: $10.1039 / b 923101 c$
}

\begin{abstract}
We report a simple methodology to fabricate PDMS multi-layer microfluidic chips. A PDMS slab was surface-treated by trichloro $(1 \mathrm{H}, 1 \mathrm{H}, 2 \mathrm{H}, 2 \mathrm{H}$-perfluorooctyl) silane, and acts as a reusable transferring layer. Uniformity of the thickness of the patterned PDMS layer and the well-alignment could be achieved due to the transparency and proper flexibility of this transferring layer. Surface treatment results are confirmed by XPS and contact angle testing, while bonding forces between different layers were measured for better understanding of the transferring process. We have also designed and fabricated a few simple types of 3D PDMS chip, especially one consisting of 6 thin layers (each with thickness of $50 \mu \mathrm{m}$ ), to demonstrate the potential utilization of this technique. 3D fluorescence images were taken by a confocal microscope to illustrate the spatial characters of essential parts. This fabrication method is confirmed to be fast, simple, repeatable, low cost and possible to be mechanized for mass production.
\end{abstract}

\section{Introduction}

PDMS chips are commonly used in a microfluidic system to achieve the goal of lab-on-a-chip. ${ }^{1-3}$ Compared to $2 \mathrm{D}$ channels, 3D structures in PDMS can achieve more functions and have been used in many microfluidic applications, such as 3D spheroid formation, ${ }^{4}$ controlled double emulsification ${ }^{5}$ and microfluidic mixer. ${ }^{6}$ 3D microfluidic networks could be used to solve computationally hard problems. $^{7}$ Also, HepG2 cells were successfully cultured in a plate-type PDMS scaffold with 3D ordered microstructure to allow cells to attach at a high density and simulated in vivo oxygen supply. ${ }^{8}$

On the other hand, omnifarious methods have been discovered, such as gray-scale lithography, ${ }^{9}$ microcontact printing with elastomeric stamps $^{10}{ }^{\mathbf{1 0}}$ microstereolithography technology, ${ }^{\mathbf{1 1}}$ stacking layers of patterned paper and double-sided adhesive tape. ${ }^{12}$ For PDMS chip, most 3D structure was fabricated by an add-on process, which stacks 2D patterned PDMS thin layers together. A sandwich molding configuration with the help of many different assisting layers was developed to realize thin layers with openings and to stack them together; ${ }^{13}$ the "membrane sandwich" method was brought forward to suggest the structure of the final system by the help of two-level photolithography; ${ }^{14}$ some researchers also tried to cut the channels of interest into long strips with cross-section about $0.5 \mathrm{~mm} \times$ $0.5 \mathrm{~mm}$ and formed into $3 \mathrm{D}$ by bending, ${ }^{15}$ while a perforated membrane method was proposed recently where photoresist posts were manually pulled out after patterned thin PDMS layer was solidified. ${ }^{16}$ In our method, the add-on process was adopted,

${ }^{a}$ Nano Science and Technology Program and KAUST-HKUST Micro/Nanofluidic Joint Laboratory, The Hong Kong University of Science and Technology, Clear Water Bay, Kowloon, Hong Kong. E-mail: phwen@ust.hk; Fax: +852 2358 1652; Tel: +852 23587979

${ }^{b}$ Department of Physics, The Hong Kong University of Science and Technology, Clear Water Bay, Kowloon, Hong Kong but what is special is that PDMS itself with surface treatment is used as a transferring layer, resulting in the uniformity of the thin layer thickness and good alignment. And the surface treatment endows the PDMS transferring layer with modified affinity to the transferred layer, makes both the bonding and peeling off process more reliable and avoids some disadvantages in the methods mentioned above, such as configuration of complex sandwich molding, two-level photolithography, handling tiny structure less then $1 \mathrm{~mm}$ or damage of master.

\section{Experimental}

The fabrication process is schematically demonstrated in Fig. 1. All the masters for each layer could be fabricated by standard photolithography. In our experiments, we used negative photoresist SU8 as the master layer and $\mathrm{Si}$ wafer as a substrate. The fabrication processing steps can be described as seven steps which are described as follows: Step 1: as can be seen from the drawing, a piece of polydimethylsiloxane (PDMS) layer (about $2 \mathrm{~mm}$ in thickness) is produced with Sylgard 184 silicone elastomer mixture (Dow Corning Corporation, Miland, USA) at a weight ratio of Base : Curing agent $=10: 1$. After solidification, it was treated by trichloro $(1 \mathrm{H}, 1 \mathrm{H}, 2 \mathrm{H}, 2 \mathrm{H}$-perfluorooctyl) silane ( $97 \%$, Sigma-Aldrich, USA), which is also called PFOCTS. The treatment was carried out in an encapsulated chamber under a pressure of about -0.5 bar to deposit a thin layer of PFOCTS onto the surface of PDMS. The same PFOCTS treatment was performed for a SU8 mode. Step 2: PDMS gel was poured onto SU8 mode and then placed in a vacuum chamber to get rid of the bubbles. Transferring PDMS slab was then placed on it with the PFOCTC layer facing the SU8 mode. A piece of plastic was placed on it while another metal could be loaded in order to provide pressure about $20-30 \mathrm{mg} / \mathrm{mm}^{2}$ and then the whole device was then put into an oven at $60{ }^{\circ} \mathrm{C}$ for about $2 \mathrm{~h}$ to solidify PDMS gel, as can be seen in step 3. After releasing from the SU8 mode (step 4), the two-layer bonded PDMS slab with a PFOCTS 


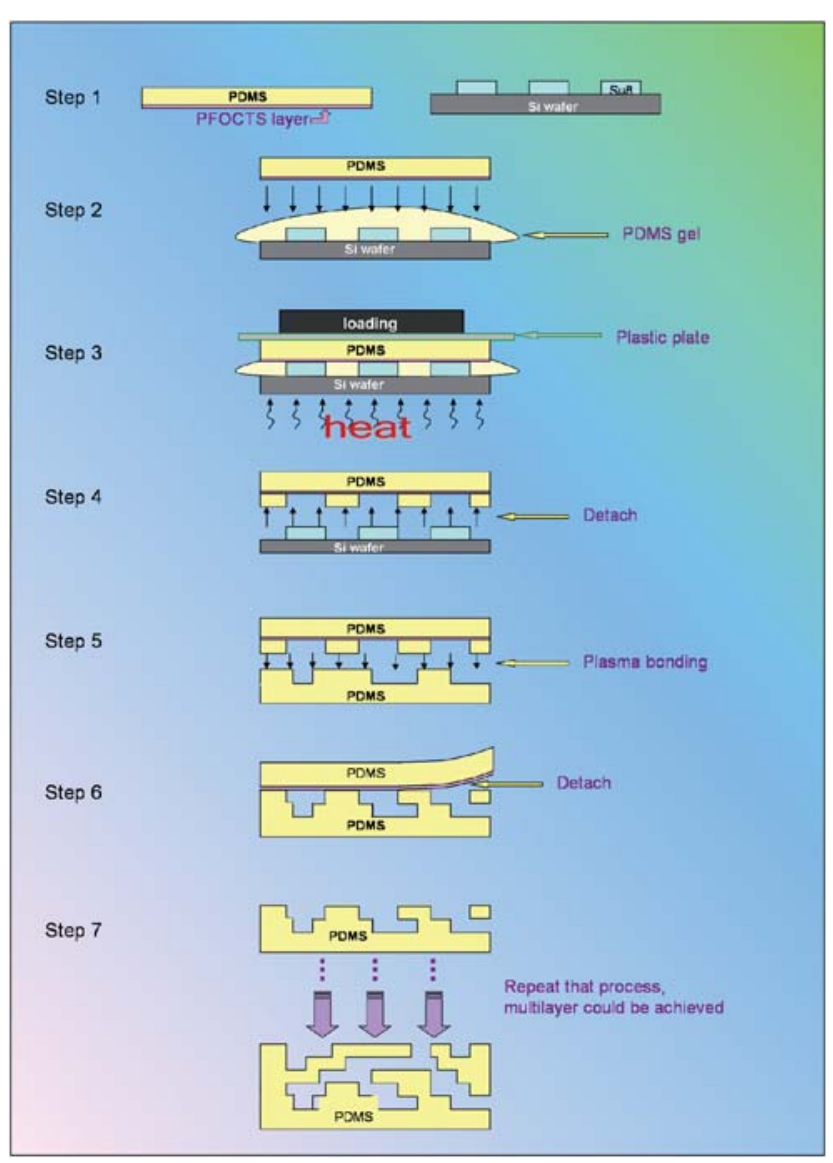

Fig. 1 Schematic view of fabrication process flow.

treated interlayer and patterned structure was ready for the next step. As can be seen from step 5 in Fig. 1, where substrate another patterned PDMS (lower one) slab and one prepared above - were put in plasma cleaner (Harrick Plasma PDC-002) to have oxygen plasma treatment for about $1 \mathrm{~min}$. Both the surfaces were covered with methanol, put together face to face and aligned under a microscope. Then, the transferring slab could be taken off, leaving the patterned thin layer well linked to the PDMS substrate, as shown in step 6. The multi-layer structure could be easily obtained by repeating this process to stack many thin patterned PDMS layers together (step 7).

\section{Results and discussion}

\section{PDMS surface treatment and characterization}

The transferring layer is the most important aspect of this methodology. First, for effective alignment, it should be transparent. Second, in order to achieve proper touch with the surface of the master without much deformation, and thereby to impart uniformity to the thickness of the thin patterned PDMS layer, the transferring layer must be sufficiently flexible. Third, the bonding force between the transferring and transferred layer should be higher than that between the transferred layer and the master, so that detachment can easily be achieved in step 4, and lower than the oxygen-plasma bonding between the two PDMS layers, in order to detach the transferring layer in step 6.
Many transferring-layer materials have been tested, such as PMMA plate, various kinds of plastic thin film (parafilm, plastic tape, wrapping paper, etc.), glass slides and others. Finally, we focused instead on PDMS, on the basis of its easy molding characteristics, transparency and flexibility. Moreover, it had already been used in the transferring layer, specifically in the fabrication of 3D-structured photocurable polymers. ${ }^{17}$ The problem with using pure bulk PDMS is that, whereas PDMS gel solidifies on its surface, strong adhesion forms at the interface, and this adhesion is often stronger than the plasma bonding between the two PDMS layers. ${ }^{18}$ To lower this bonding force, PFOCTS was employed. This chemical is commonly used as a self-assembled monolayer (SAM) on a mold for releasing as an anti-sticking layer for nanoimprint. ${ }^{19}$ PFOCTS has several fluorin terminates, which are very inert and repel water and other organic substances. ${ }^{20}$

To ensure that the PFOCTS was deposited onto the surface of the transferring PDMS layer, the surface was characterized by means of XPS spectra (X-ray photoelectron spectroscopy, PHI 5600, Physical Electronics Corp.) for elemental composition analysis and a contact angle analyzer for hydrophobicity. The results are shown in Table 1 . After the surface treatment, the F concentration increased from almost 0 to about $0.39 \%$. As only PFOCTS contains F, we could conclude that there should be one layer of PFOCTS on the surface of the PDMS. This surface modification was confirmed also by the contact-angle testing: the approximate $89^{\circ}$ contact angle of a pure PDMS surface was increased to $98^{\circ}$ owing to the hydrophobicity of PFOCTS.

The effect of the surface treatment was tested by measuring the bonding forces between different layers shown in Fig. 2(a): the pure bulk PDMS as substrate (layer 1), the pure PDMS thin layer (layer 2), and the surface-treated transferring PDMS slab (layer 3). Layers 1 and 2 were combined using the plasma bonding method $^{21}$ by which the two bonding surfaces are treated with oxygen plasma for about $1 \mathrm{~min}$, after which they are put together to initiate chemical bonding. Layers 2 and 3 were combined by PDMS half-cure bonding. The bonding force testing was conducted by means of a method similar to that which had already been reported. ${ }^{18} \mathrm{~A}$ hole of $1 \mathrm{~mm}$ diameter was formed in the interface, through which was applied pressure, which was monitored using the digital pressure sensor 'Druck DPI 104' (GE Druck, Leicester, UK). The pressure value adequate to break the adhesion, as recorded, is shown in Fig. 2(b). The plasma bonding force was around $200 \mathrm{kPa}$, which is consistent with the results in that previous paper. Additionally, the half-cure bonding force was reduced from around $600 \mathrm{kPa}$ to about $50 \mathrm{kPa}$, owing to the PFOCTS layer. The weaker bonding force enables the structured PDMS thin layer to be transferred from the transferring layer to the PDMS substrate by plasma bonding.

Table 1 XPS results and contact angle

\begin{tabular}{lllllll}
\hline & \multicolumn{4}{l}{ Atomic concentration } & \\
\cline { 2 - 5 } & $\mathrm{C}$ & $\mathrm{O}$ & $\mathrm{F}$ & $\mathrm{Si}$ & $\begin{array}{l}\text { Contact } \\
\text { angle }\end{array}$ \\
\hline Pure PDMS & 46.53 & 28.57 & 0.01 & 24.89 & $89^{\circ}$ \\
PDMS with surface treatment & 45.01 & 28.81 & 0.39 & 25.79 & $98^{\circ}$ \\
\hline
\end{tabular}




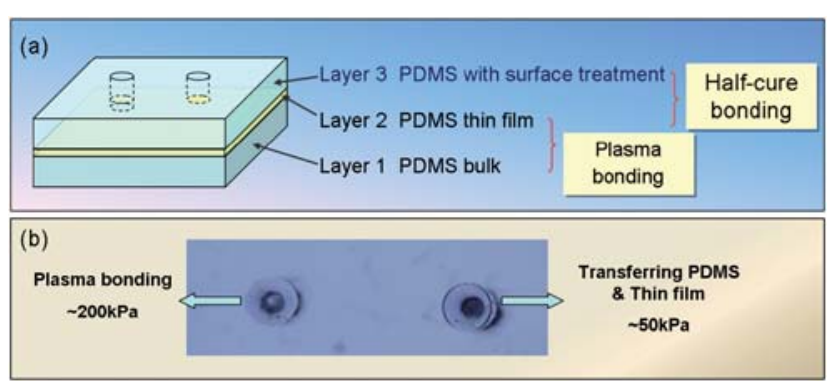

Fig. 2 Sketch map of bonding strength testing. (a) Illustration of layers and holes, (b) snapshot of testing holes.

We noticed also that the contact angle was not very large, and the bonding force between the PFOCTS-treated PDMS and the patterned PDMS thin layer was higher than the one between PFOCTS-treated Si/SU8 master and that patterned thin layer. One possible reason is that PDMS is porous, ${ }^{22}$ and so there were some pores on the surface of the transferring PDMS slab. Though PFOCTS prevents the bonding of uncured PDMS gel with a cured PDMS slab, some un-crosslinked PDMS molecules might yet diffuse into the cured PDMS slab by those pores, solidify, and form some link between them. Also, the transferring PDMS slab is found to be reusable without retreatment by PFOCTS.

\section{D PDMS chips}

In $3 \mathrm{D}$ channels, the most fundamental aspect is the cross area, in which the two channels cross without forming any junction.

Fig. 3 illustrates the fabrication method. The main channels are put on the two side layers while the junction layer is located in between (Fig. 3(a)). In this structure, the crossing channels go to

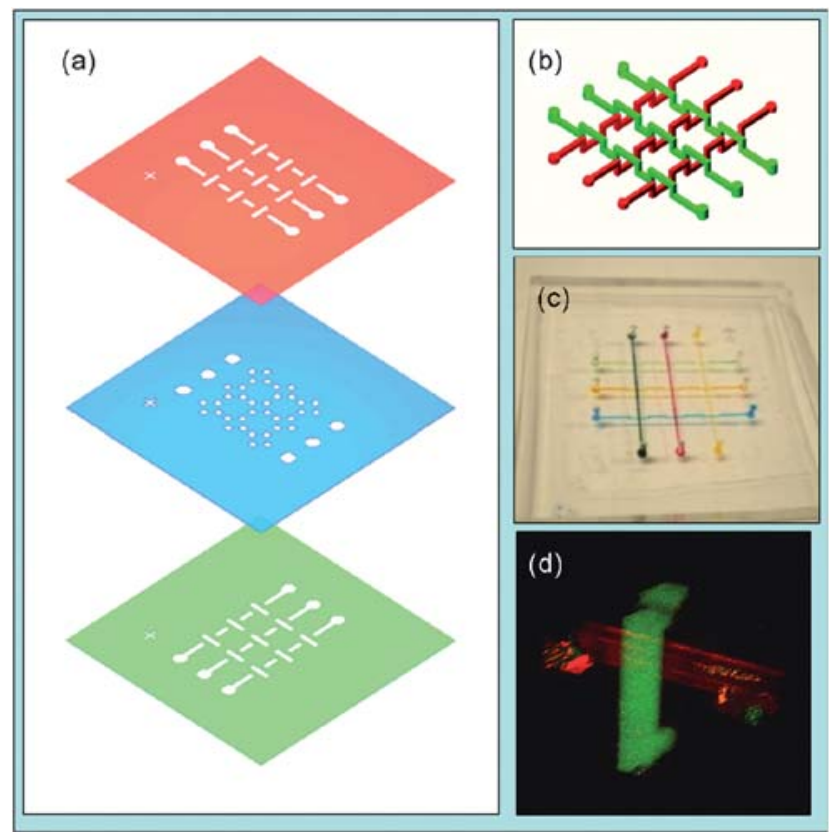

Fig. 3 Design for the basic cross part for 3D structure: (a) schematic view of each layer, (b) whole view, (c) optic images and (d) fluorescent image taken by confocal microscope. different layers to avoid encountering each other in this cross area. Fig. 3(a) is a schematic view of each layer of a demonstration chip. For this chip, we could bond these 3 layers together using the fabrication method described previously in this paper. The result is illustrated in Fig. 3(b), and optical images of the channels, which were filled with colored water, are shown in Fig. 3(c). Utilizing fluids dyed with different fluorescent colors, confocal microscopy was used to closely examine the cross area, and reconstruction of the 3D image is shown in Fig. 3(d). In this image, the two channels cross each other without connecting, by flowing to different layers.

3D PDMS chip fabrication has been investigated by many researchers. Whereas structures of 4 layers or fewer are easily obtained by two-level photolithography, but multi-layer structures, especially those of more than 5 layers, are not so readily achieved. Fig. 4 illustrates a simple structure consisting of 5 layers. In Fig. 4(a), schematic view, layers 1, 3 and 5 are the main flow channels, layers 2 and 4 being the connection layers in the cross area. The small patterns in the middle of each layer are for alignment. In the optical image and schematic view of the final 3D structure shown in Fig. 4(b) and (e), respectively, the yellow and blue channels can be seen going to layers 1 and 3 alternatively with the 3 cross areas and, finally, going together, at the top point, into layer 5 , where they mix, the color turning to green, before flowing out. Fig. 4(c) and (d) are fluorescent images of some of the essential components. The fluids were dyed fluorescent red (Rhodamine: Rhodamine B isothiocyanate, Sigma Aldrich) or green (FITC: Fluorescein isothiocyanate-dextran, $4 \mathrm{kD}$, Sigma Aldrich), so that when they mixed, the color yellow would appear. As is seen in Fig. 4(c), the two fluids arrive at the connecting point and flow together into another channel. The yellow color in this image shows that the mixing begins at the interface of the two flows. When it goes to the cross area nearest to the outlet, as shown in Fig. 4(d), the yellow color in the mixing channel becomes more homogeneous and the interface could not be recognized, which indicates that the mixing result is not bad. It is apparent also that the red color is more concentrated at the wall of the channel, owing to the fact that PDMS is porous and as such, easily absorbs small molecules such as Rhodamine. ${ }^{23}$
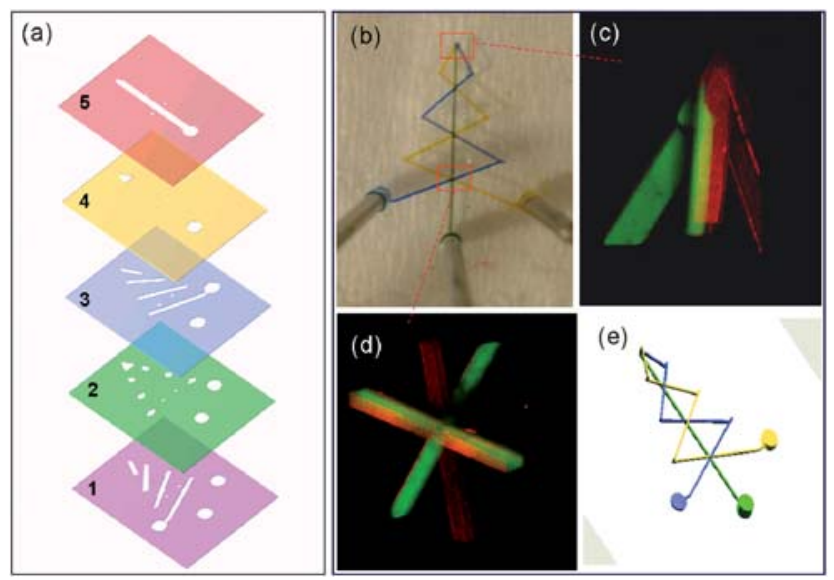

Fig. 4 A five layer 3D structure: (a) illustration of patterns in each layer, (b) optical image, (c), (d) 3D fluorescent image by confocal microscope of two essential areas, (e) schematic view of whole structure. 

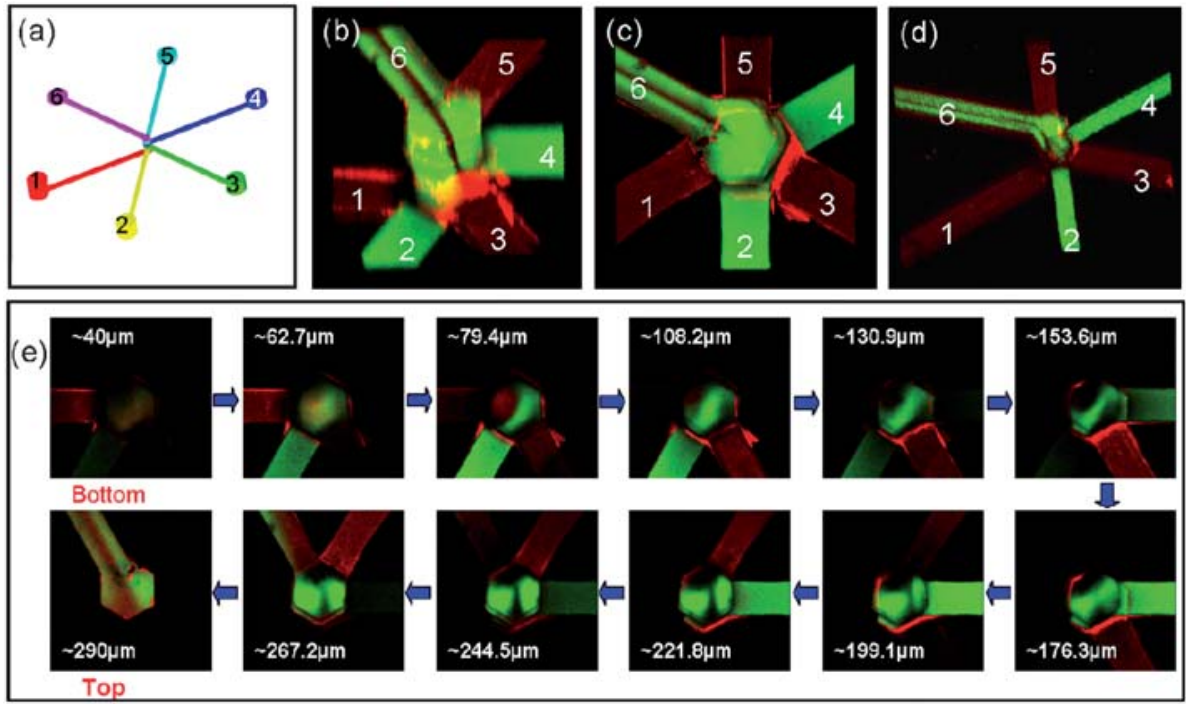

Fig. 5 A six layer structure with common junction for fluid mixing and flow pattern observation: (a) schematic view of the whole structure, (b)-(d) fluorescent images of junction area at different view point, (e) fluorescent images taken at different levels.

Finally, as shown in Fig. 5, we fabricated a 6-layer structure to exhibit the power of this fabrication process. Here, 5 channels flowing in 5 different layers, come to the common junction area in the middle, and flow out together through the 6th layer. The schematic view of this structure is illustrated in Fig. 5(a), while fluorescent images from different points of view of the junction area are shown in Fig. 5(b)-(d). We filled channels 1, 3, and 5 with Rhodamine solution and channels 2 and 4 with FITC. The channel was about $100 \mu \mathrm{m}$ wide and $50 \mu \mathrm{m}$ high. Fig. 5(e) includes images scanned layer-by-layer by confocal microscopy. In each image, the distance from the scanned layer to the bottom of the lowest channel is marked. From the bottom to the top, the channels appear one by one, and the change of flow pattern in the junction area also could be observed. Though the absorption of Rhodamine by PDMS is a defect, 3D flow pattern could still benefit from this 3D PDMS fabrication. Here it is seen clearly that 6 patterned PDMS layers, as thin as $50 \mu \mathrm{m}$, could be easily integrated together with good alignment. This $3 \mathrm{D}$ multilayer PDMS channel fabrication, together with confocal microscopy, can be applied to and give great help in many lab-on-a-chip processes, especially in biology application such as DNA and protein testing, cell culture and on-chip observation.

\section{Conclusions}

In this paper, we illustrate a simple method of fabricating 3D PDMS chips by an add-on process. A PFOCTS-treated PDMS slab used as transferring layer exhibited a proper adhesive force as bonded with a patterned PDMS thin layer. Moreover, good alignment could be achieved in the oxygen-plasma bonding. Some basic 3D structures were achieved, and 3D images were taken by confocal microscopy. As only the master layer (a $\mathrm{Si}$ wafer and SU8 or other material), PDMS and PFOCTS, are required, fast fabrication and even mass production are achievable. Many chemical reaction observations and biological applications in microfluids would benefit from this fast and simple 3D fabrication method.

\section{Acknowledgements}

This publication is based on work partially supported by Award No. SA-C0040/UK-C0016, made by King Abdullah University of Science and Technology (KAUST), Hong Kong RGC grants HKUST 603608 . The work was also partially supported by the Nanoscience and Nanotechnology Program at HKUST. The authors would like to thank Dr Jiaxing Li and Mr Xiuqing Gong, who gave some help and good suggestion in material choosing, characterization and analyse.

\section{References}

1 C. Liu, Adv. Mater., 2007, 19, 3783-3790.

2 J. De Jong, R. G. H. Lammertink and M. Wessling, Lab Chip, 2006, 6, $1125-1139$.

3 S. K. Sia and G. M. Whitesides, Electrophoresis, 2003, 24, 3563-3576.

4 H. Ota, K. Deguchi, S. Kita, R. Yamamoto and N. Miki, 2008 International Symposium on Micro-NanoMechatronics and Human Science, MHS 2008, 2008, pp. 202-207.

5 F. Chang and Y. Su, J. Micromech. Microeng., 2008, 18, 065018.

6 D. Therriault, S. R. White and J. A. Lewis, Nat. Mater., 2003, 2, 265 271.

7 D. T. Chiu, E. Pezzoli, H. Wu, A. D. Stroock and G. M. Whitesides, Proc. Natl. Acad. Sci. U. S. A., 2001, 98, 2961-2966.

8 C. Provin, K. Takano, T. Yoshida, Y. Sakai, T. Fujii and R. Shirakashi, Biomed. Microdevices, 2009, 11, 485-494.

9 Y. Oppliger, P. Sixt, J. M. Stauffer, J. M. Mayor, P. Regnault and G. Voirin, Microelectron. Eng., 1994, 23, 449-454.

10 X. Zhao, Y. Xia and G. M. Whitesides, Adv. Mater., 1996, 8, 837-840.

11 H. Kang, I. H. Lee and D. Cho, J. Manuf. Sci. Eng., 2004, 126, $766-$ 771.

12 A. W. Martinez, S. T. Phillips and G. M. Whitesides, Proc. Natl. Acad. Sci. U. S. A., 2008, 105, 19606-19611.

13 B. Jo, L. M. Van Lerberghe, K. M. Motsegood and D. J. Beebe, J. Microelectromech. Syst., 2000, 9, 76-81, DOI: 10.1109/84.825780.

14 J. R. Anderson, D. T. Chiu, R. J. Jackman, O. Chemiavskaya, J. C. McDonald, H. Wu, S. H. Whitesides and G. M. Whitesides, Anal. Chem., 2000, 72, 3158-3164. 
15 H. Wu, T. W. Odom, D. T. Chiu and G. M. Whitesides, J. Am. Chem. Soc., 2003, 125, 554-559.

16 Y. Luo and R. N. Zare, Lab Chip, 2008, 8, 1688-1694.

17 D. Bartolo, G. Degré, P. Nghe and V. Studer, Lab Chip, 2008, 8, 274 279.

18 M. A. Eddings, M. A. Johnson and B. K. Gale, J. Micromech Microeng., 2008, 18, 067001.
19 J. Chen, F. Ko, K. Hsieh, C. Chou and F. Chang, J. Vac. Sci. Technol., B, 2004, 22, 3233-3241.

20 D. M. Lemal, J. Org. Chem., 2004, 69, 1-11.

21 J. J. McMahon, Y. Kwon, J. Lu, T. S. Cale and R. J. Gutmann, Mater Res. Soc. Symp. Proc., 2003, 795, 99-104.

22 M. W. Toepke and D. J. Beebe, Lab Chip, 2006, 6, 1484-1486.

23 R. Mukhopadhyay, Anal. Chem., 2007, 79, 3249-3253. 